\title{
Spatial inhomogeneity in spectra and exciton dynamics in porphyrin micro-rods and micro-brushes: Confocal microscopy
}

\author{
SHYAMTANU CHATTORAJ and KANKAN BHATTACHARYYA* \\ Department of Physical Chemistry, Indian Association for The Cultivation of Science, Jadavpur, \\ Kolkata 700 032, India \\ e-mail: pckb@iacs.res.in
}

MS received 2 July 2016; revised 12 August 2016; accepted 13 August 2016

\begin{abstract}
In an aqueous acidic solution, the porphyrin meso-tetra(4-sulfonatophenyl) porphyrin tetrasodium salt (TPPS) forms different kinds of assembly (micro-rods and micro-brush) depending on condition of evaporation. The exciton dynamics and emission spectra of the micro-rods and micro-brushes depend on spatial inhomogeneity. This is elucidated by time-resolved confocal microscopy.
\end{abstract}

Keywords. Porphyrin; micro-rod; anisotropy; exciton coupling; confocal microscopy.

\section{Introduction}

Supra-molecular assemblies of porphyrin play a central role in light harvesting during photosynthesis. ${ }^{1-10}$ In such a system, the absorbed photon shuttles between different units giving rise to energy transfer and exciton coupling. This results in highly efficient and irreversible energy transfer to the reaction centre. Among these supramolecular assemblies, tubular/rod-like aggregate is one of the promising candidate for artificial/model light harvesting system. ${ }^{11-14}$ Such tubular/rod-like structures are formed via self-assembly of conjugated dye molecules.

Porphyrins are known to form different kinds of aggregates such as nanotube aggregates/rod-like structures under highly acidic conditions and in the presence of other additives. ${ }^{15-23}$ The nanotubular aggregate exhibits a sharp red shifted absorption band characteristic of J-aggregates. Such J-aggregates of porphyrins may exhibit exciton dynamics similar to those observed in a conjugated polymer, ${ }^{24}$ and in carbon nanotubes. ${ }^{25} \mathrm{In}$ bulk solutions, the exciton dynamics in such supramolecular aggregates has been studied in a number of ways. Scholes and co-workers studied coherent intrachain electronic energy transfer between different segments of a conjugated polymer using two-dimensional photon echo (2DPE) experiments. ${ }^{24}$ Fleming and coworkers used two-color electronic coherence photon

\footnotetext{
*For correspondence

Dedicated to Professor T K Chandrasekhar on the occasion of his $60^{\text {th }}$ birth anniversary
}

echo experiment (2CECPE) to investigate role of protein environment in electronic coherence in photosynthetic complexes. ${ }^{1}$ Huang and co-workers applied ultrafast optical spectroscopy to probe time and temperature dependent exciton dynamics of an tubular porphyrin aggregate. They have also invoked stochastic exciton modeling to explain dynamics of such Frenkel excitons. ${ }^{20}$

In contrast to the bulk/ensemble studies, there are relatively few single molecule studies on the exciton dynamics of such supramolecular aggregates. Weisman and co-workers have used single molecule microscopy to probe space dependent exciton dynamics of a single carbon nano-tube. ${ }^{25}$ In this case, the exciting laser is focused on different parts of a carbon nano-tube (CNT) immobilized on a glass slide. They have estimated the exciton diffusion length and diffusion coefficient from the analysis of step amplitudes in the single molecule time trace of CNT. Köhler and co-workers used polarization-resolved fluorescence excitation spectroscopy to record excitation spectra of individual chlorosomes from the photosynthetic green sulfur bacterium. ${ }^{26}$ They reported significant difference in the excitation spectra of different single cholorosomes and attributed this to the variations of the excitonic compositions of the $\mathrm{BChl}$ $\mathrm{c}$ aggregates present in the cholorosomes.

In the present work, we have employed time resolved confocal microscopy to study the exciton dynamics and polarization properties of a micro-tubular aggregates derived from a porphyrin meso-tetra(4-sulfonatophenyl) porphyrin (TPPS). These micro-tubular aggregates are formed via ionic self assembly of porphyrin monomers in highly acidic condition $(\mathrm{pH}=1)$. These 
supramolecular structures are devoid of any kind of protein scaffold and faithfully represents a biomimetic reminiscent of the chlorosomes of green sulfur bacteria. ${ }^{26-29}$ These tubular/rod like structures are of different dimensions and grow upto several microns.

Considering the immense importance of this supramolecular aggregates in artificial light harvesting system it is important to elucidate the exciton dynamics of individual micro-rods which can be achieved by using confocal microscopy and polarization resolved single molecule fluorescence spectroscopy. ${ }^{30-41}$ In the present work, we have studied exciton dynamics of two different porphyrin suprastructure (prepared under two different conditions) and monitored fluctuation in fluorescence intensity in them. We have also studied the polarization properties of such micro-structures from single molecule anisotropy and observed marked difference in time dependence of steady state anisotropy. We will show that the polarization properties of porphyrin micro-rods and micro-brush are significantly different. To the best of our knowledge, the micro-brush structure of TPPS has been reported here for the first time.

\section{Experimental}

\subsection{Materials}

Meso-tetra(4-sulfonatophenyl) porphyrin tetrasodium salt (TPPS, Sigma), hydrochloric acid (Fischer Scientific) were used as received.

\subsection{Methods}

2.2a Preparation of TPPS micro-rod: A stock solution of TPPS was prepared in HPLC grade deionized water. The micro-tubular/rod-like structures were prepared by adding concentrated $\mathrm{HCl}$ to the stock solution. The appropriate concentration of $\mathrm{HCl}$ was maintained at $0.05 \mathrm{M}$ to maintain a highly acidic $\mathrm{pH}(\mathrm{pH}=1)$. The supramolecular structures were allowed to grow for 8 hours from an aqueous acidic solution of TPPS $(2 \mu \mathrm{M})$. The concentration of the monomer solution at $\mathrm{pH}=4$ was also kept at $2 \mu \mathrm{M}$ for comparison.

2.2b Steady-state absorption and fluorescence spectroscopy: The steady-state absorption and fluorescence spectra were recorded with a Shimadzu UV-2401 spectrophotometer and Spex FluoroMax-3 spectro-fluorimeter, respectively.

2.2c Experimental set-up for confocal microscopy: The experimental set-up for confocal microscopy has been described in our previous publications. ${ }^{40,41}$ To summarize, a combination of confocal microscope (Olympus IX-71) and TCSPC setup (PicoQuant, MicroTime 200, numerical aperture $(\mathrm{NA}) \approx 1.2$ ) and a pulsed picosecond diode laser (PDL 828-S "SEPIA II," Pico Quant, at $470 \mathrm{~nm}$ ) were used. The diffraction limited spot size is $0.6 \lambda / 1.2 \sim \lambda / 2$. Laser power used was $\sim 10 \mu \mathrm{W}$. Polarization resolved fluorescence intensity with different polarizations $\left(\mathrm{I}_{\|}\right.$, and $\mathrm{I}_{\perp}$ ) were separated using a polarizer cube (Chroma) and were detected by two separate detectors (micro photon device, MPD). Appropriate narrow band-pass filters were used (e.g., XBPA720, 690, etc. Asahi Spectra) to collect TCSPC decay at specific emission wavelengths. The fluorescence decays at magic angle conditions can be represented as follows,

$$
\begin{aligned}
I_{\text {magic }}(t) & =I_{\amalg}(t) \cos ^{2}\left(54.75^{\circ}\right)+I_{\perp}(t) \cdot G \cdot \sin ^{2}\left(54.75^{\circ}\right) \\
& =(1 / 3) I_{\amalg}(t)+(2 / 3) \cdot G \cdot I_{\perp}(t)
\end{aligned}
$$

$\mathrm{G}$ factor for this microscope setup was found to be $\sim 1.2$. $^{41}$

2.2c1 Fluorescence anisotropy measurement: The anisotropy function, $\mathrm{r}(\mathrm{t})$, was obtained using the formula,

$$
r(t)=\frac{I_{\amalg}-G I_{\perp}}{I_{\amalg}+2 G I_{\perp}}
$$

where $I_{\|}$and $I_{\perp}$ are fluorescence intensity in the parallel and perpendicular polarization. These are obtained from the polarization resolved fluorescence intensity trajectories of TPPS superstructures recorded in the confocal microscope.

2.2c2 Emission spectra: The emission spectra of TPPS supramolecular structures were recorded using an electron multiplying charge-coupled device (EMCCD, ANDOR Technology) attached to a spectrograph (ANDOR Technology, Shamrock series). ${ }^{40,41}$

2.2c3 Fluorescence lifetime measurement: For recording instrument response function (IRF), we used a bare slide and collected the scattered laser light. The FWHM of the IRF for excitation at $470 \mathrm{~nm}$ is $\sim 70 \mathrm{ps}$. The fluorescence decay is deconvoluted using the IRF and DAS6 v6.3 software.

\section{Results and discussion}

\subsection{Steady state absorption and emission spectra}

Figure 1a shows steady state absorption and emission spectra of TPPS monomer in aqueous $\mathrm{HCl}$ at $\mathrm{pH}=4$. 


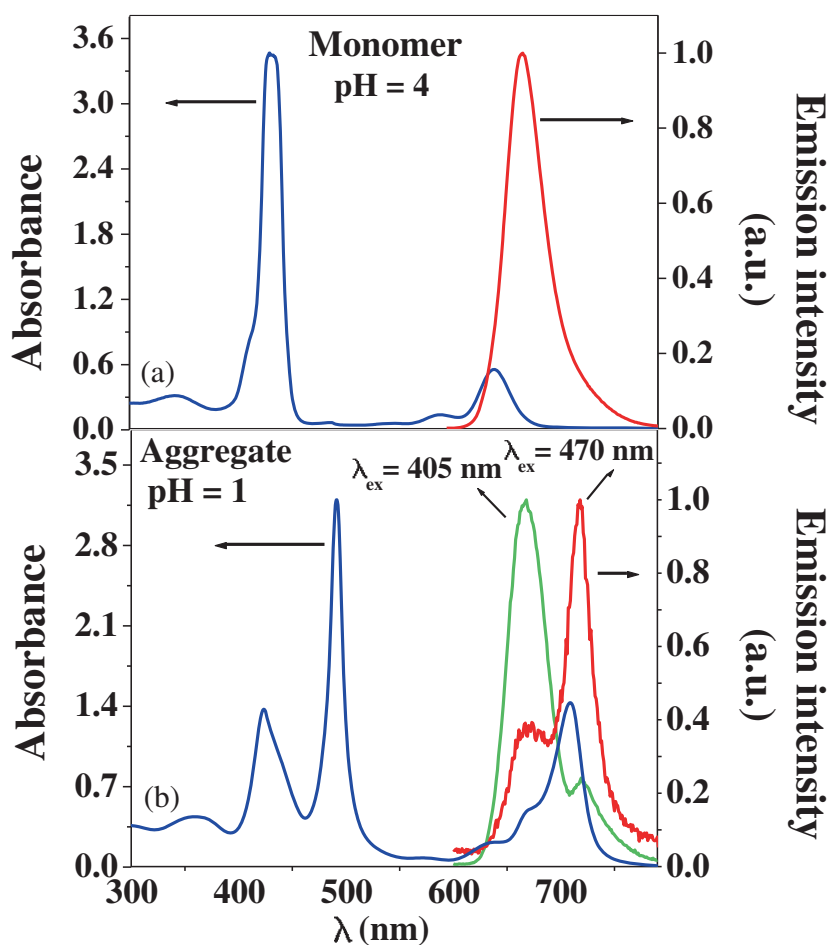

Figure 1. Steady state absorption and emission spectra of TPPS monomer at $\mathrm{pH}=4$ (upper panel) and TPPS aggregate at $\mathrm{pH}=1$ (lower panel). Concentration of the solution is 2 $\mu \mathrm{M}$. Path-length $=1 \mathrm{~cm}$.

At $\mathrm{pH}=4$, TPPS exists as a diacid form and exhibits an absorption peak at $430 \mathrm{~nm}$ and $650 \mathrm{~nm}$ with no absorption peak at 490 or $700 \mathrm{~nm}$. The monomer (diacid) form exhibits an emission maximum $\sim 672 \mathrm{~nm}$. The position of the emission maximum for monomer form does not change with different excitation wavelengths.

Figure $1 \mathrm{~b}$ shows steady state absorption and emission spectra of TPPS aggregate at $\mathrm{pH}=1$. At $\mathrm{pH}=1$ the main absorption peak is red shifted to a strong narrow peak at $490 \mathrm{~nm}$ with a weak blue shifted peak at $424 \mathrm{~nm}$. In addition, there is a new peak at $\sim 700 \mathrm{~nm}$. The width of the $490 \mathrm{~nm}$ is extremely narrow due to exciton coupling. At $\mathrm{pH}=1$, TPPS is known to form rod like structure due to self assembly of TPPS molecules. ${ }^{20}$ This results in an extremely narrow peak at $490 \mathrm{~nm}$ due to exciton coupling. In addition at $\mathrm{pH}=1$, TPPS exhibits an emission peak of $668 \mathrm{~nm}$ and $720 \mathrm{~nm}$ when excited at $405 \mathrm{~nm}$ and $470 \mathrm{~nm}$, respectively. The previous peak is similar to the monomer form while the later peak corresponds to the aggregate.

\subsection{Confocal image of TPPS microstructures}

Figure 2 shows the confocal images of TPPS micro structures formed at $\mathrm{pH}=1$. It is readily seen that a micro-brush like structure is formed (Figure 2a) when a drop of an aqueous $\mathrm{HCl}$ solution $(\mathrm{pH}=1)$ of TPPS is taken on a stationary glass surface and allowed to evaporate slowly. These brush like structure are very long $(\sim 10-40 \mu \mathrm{m})$. These are expected to originate from the self assembly of TPPS from the slowly evaporating solution, at $\mathrm{pH}=1$.

Figure $2 \mathrm{~b}$ shows confocal image of porphyrin micro-rods. These micro-rods are formed by spin-cast method. ${ }^{42-44}$ In this case, a drop of an aqueous $\mathrm{HCl}$ solution $(\mathrm{pH}=1)$ of TPPS is taken on a glass slide and then it was spun rapidly. The length of the rods varies from $1 \mu \mathrm{m}$ to $10 \mu \mathrm{m}$. Spin-casting method has been used earlier to prepare different kinds of porphyrin assemblies (e.g., micro-scale and nanoscale rings, rods, and fibers). ${ }^{42-44}$ In the present study, the morphology of the rods and the micro-brush are distinctly different.

\subsection{Emission spectra of TPPS micro-structures recorded under a confocal microscope}

Figure 3a shows emission spectra of TPPS micro-rod recorded under a confocal microscope. The shape of the
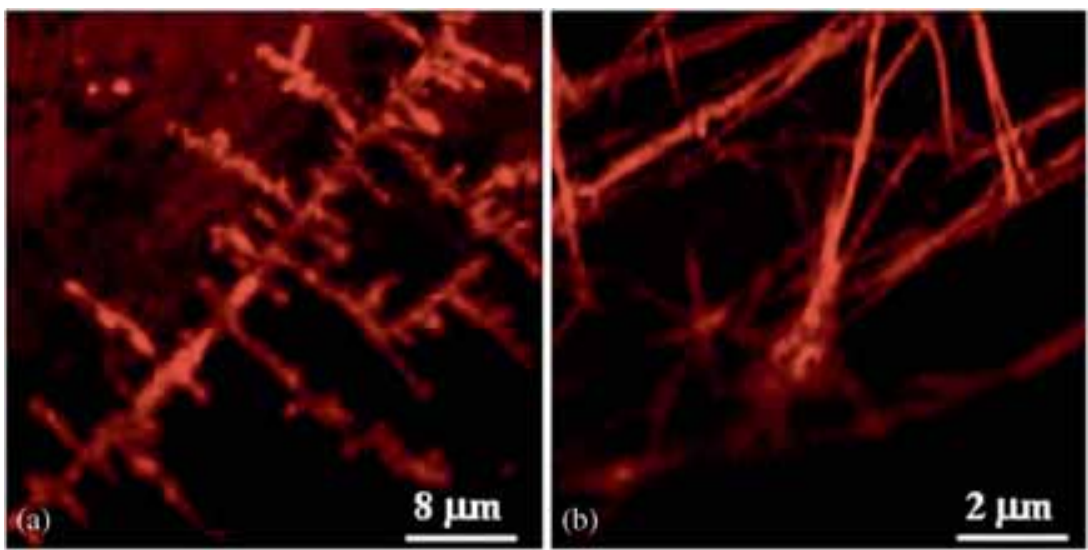

Figure 2. Confocal microscopic images of TPPS micro-structures: (a) porphyrin micro-brush. (b) Porphyrin micro rods. $\lambda_{\mathrm{ex}}=470 \mathrm{~nm}$. 

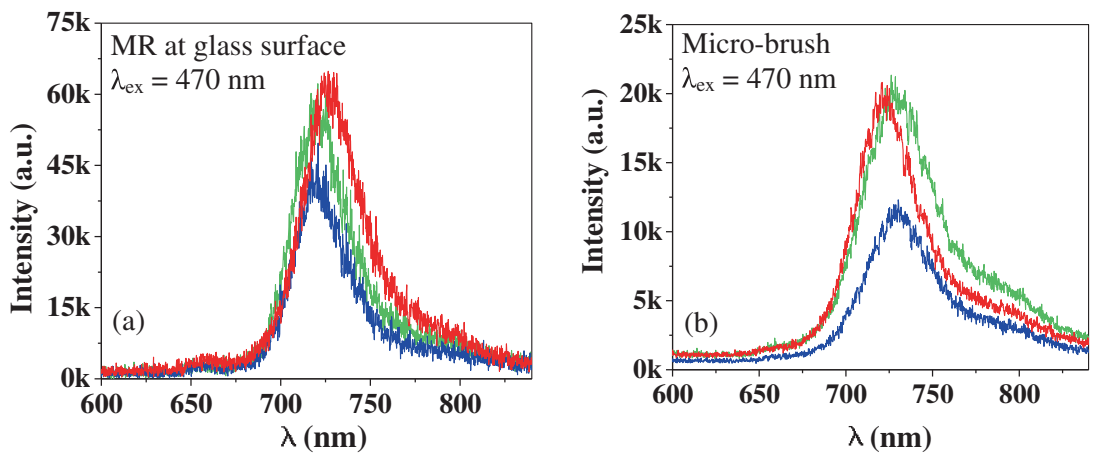

Figure 3. Emission spectra of TPPS micro-structures recorded under a confocal microscope: (a) Porphyrin micro-rod at $\lambda \mathrm{ex}=470 \mathrm{~nm}$. (b) Porphyrin micro-brush at $\lambda$ ex $=470 \mathrm{~nm}$. The different colors correspond to different positions in the TPPS micro-structures.

emission spectra is Lorenzian in nature. The position of the emission maxima does not change with different excitation wavelengths. It may be noted that the spatial resolution of our confocal microscope is $200 \mathrm{~nm}$. Thus, we are able to record emission spectra from a very small space.

The emission maximum varies from $720 \mathrm{~nm}$ to 727 $\mathrm{nm}$ for different micro-rods and different positions of a single micro-rod. The FWHM of the emission spectra also shows a distribution from $450-850 \mathrm{~cm}^{-1}$. This indicates local heterogeneity or presence of trapped states in the micro-rods which results in different emission maxima and emission line widths.

TPPS micro-brush also exhibits similar behaviour (Figure 3b). In this case, at different positions, emission maxima vary from $720 \mathrm{~nm}$ to $728 \mathrm{~nm}$ and FWHM varies from 900 to $1200 \mathrm{~cm}^{-1}$ for different positions of TPPS micro-brush. The higher FWHM of microbrushes is consistent with an ordered structure with increased intermolecular interactions.

\subsection{Fluorescence decay of TPPS micro-structures}

Figure 4a shows the fluorescence decay of TPPS monomer in aqueous $\mathrm{HCl}$ solution at $\mathrm{pH}=4$ and TPPS micro-rod at $\mathrm{pH}=1$. The fluorescence decay $\left(\lambda_{\text {em }}=\right.$ $660 \mathrm{~nm}$ ) of TPPS monomer in $\mathrm{pH}=4$ is single exponential and exhibits a fluorescence lifetime of $3.2 \mathrm{~ns}$. Interestingly, the fluorescence decay $\left(\lambda_{\mathrm{em}}=720 \mathrm{~nm}\right)$ of TPPS micro-rod in $\mathrm{pH}=1$ is tri-exponential in nature and exhibits a fast component of $70 \mathrm{ps,} \mathrm{an} \mathrm{intermediate}$ component of 300 ps and a monomer-like long component of $3.2 \mathrm{~ns}$ (Table 1). It may be mentioned that the $720 \mathrm{~nm}$ emission band is the characteristics of microrod formation. Thus, the reduced lifetime of $70 \mathrm{ps}$ and $300 \mathrm{ps}$ in the case of MR corresponds to the excitation energy transfer due to exciton coupling. The long component of $3.2 \mathrm{~ns}$ arises from the residual monomers and not from the aggregate.

Surprisingly, the long component vanishes in the case of immobilized TPPS micro-rod and micro-brush. Both
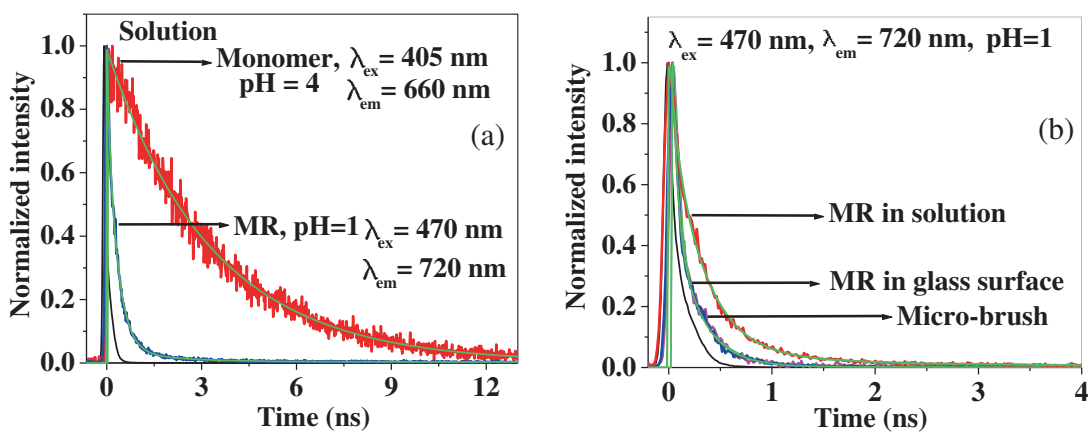

Figure 4. (a) Comparison of fluorescence decay of TPPS monomer $(\mathrm{pH}=4)$ and micro-rod $(\mathrm{MR})$ in bulk solution $(\mathrm{pH}=1)$. (b) Comparison of fluorescence decay of TPPS micro-rod in bulk solution, in glass surface and TPPS microbrush. 
Table 1. Decay parameters of fluorescence decay of TPPS micro-structures.

\begin{tabular}{lccccc}
\hline System & $\lambda_{\mathrm{ex}}$ & $\lambda_{\mathrm{em}}$ & $\tau_{1}(\mathrm{ps})\left(\mathrm{a}_{1}\right)$ & $\tau_{2}(\mathrm{ps})\left(\mathrm{a}_{2}\right)$ & $\tau_{3}(\mathrm{ps})\left(\mathrm{a}_{3}\right)$ \\
\hline Monomer in solution $(\mathrm{pH} \mathrm{4})$ & $405 \mathrm{~nm}$ & $660 \mathrm{~nm}$ & - & - & $3200(1)$ \\
Micro-rod in solution $(\mathrm{pH} 1)$ & $470 \mathrm{~nm}$ & $720 \mathrm{~nm}$ & $70(0.3)$ & $300(0.55)$ & $3200(0.15)$ \\
Micro brush & $470 \mathrm{~nm}$ & $720 \mathrm{~nm}$ & $50(0.65)$ & $250(0.35)$ & - \\
Micro rod on glass surface & $470 \mathrm{~nm}$ & $720 \mathrm{~nm}$ & $50(0.65)$ & $250(0.35)$ & - \\
\hline
\end{tabular}

of them exhibit a major fast component of 50 ps and another component of $250 \mathrm{ps}$. The fast component has a major contribution (65\%) than the other component $(35 \%)$. The average lifetime of both TPPS micro-rod and micro-brush is quite similar (120 ps) and does not depend on excitation wavelength to a great extent. This indicates that in the self-assembled micro structures of TPPS, fluorescence lifetime $(\sim 120 \mathrm{ps})$ is largely reduced compared to its diacid monomer form (3200 ps).

\subsection{Fluorescence intensity vs. time trajectories: TPPS micro-rod and micro-brush}

Figure 5a shows fluorescence intensity vs. time trajectories of TPPS micro-rod. It is readily seen that fluorescence intensity fluctuates with time for a certain time period with discrete energy changes. Such discrete changes in the fluorescence intensity in the micro-rods may be due to the presence of disordered states in this micro-structure. ${ }^{45}$ Surprisingly, the TPPS micro-brush (Figure 5b) does not exhibit such fluorescence intensity fluctuation which shows step-wise or discrete intensity changes. Note, these micro-brushes are more organized and larger in size than the micro-rods. This large size and different organization may result into the absence of such discrete energy change in the fluorescence intensity trajectory. Thus, the absence of step-wise fluctuation in the case of micro-brush reconciles existence of an ordered structure with increased intermolecular interactions.

\subsection{Fluorescence anisotropy image: TPPS micro-rod and micro-brush}

In this section we discuss the fluorescence anisotropy image of different microstructures of TPPS porphyrin. Figure $6 \mathrm{a}-\mathrm{c}$ demonstrates simultaneously collected polarization resolved fluorescence intensity image of TPPS micro-rod. It can be readily seen from Figure 6c that fluorescence is coming from both the parallel and perpendicular orientations of the transition dipoles. This shows that the transition dipoles of some of the micro-rods are orientated along the parallel orientation while the others are orientated in perpendicular direction. Even for a single micro-rod, some portion of it is oriented along the parallel while some is oriented along the perpendicular direction. This is reflected in the corresponding fluorescence anisotropy image (Figure 6d). It is readily seen from Figure $6 \mathrm{~d}$ that the fluorescence anisotropy values are different for different micro-rods as well as different part of a single micro-rod.

In contrast to the TPPS micro-rods, the micro-brush structures exhibit quiet homogeneous distribution of fluorescence anisotropy (Figure 6h). The fluorescence anisotropy does not change significantly among different micro-brushes as well as different parts of a microbrush. This indicates that the response of the transition dipoles with respect to fluorescence polarization is fundamentally different between TPPS micro-rods and micro-brush. In other words, the molecular packing of the individual TPPS segment is different in these two supramolecular structures.
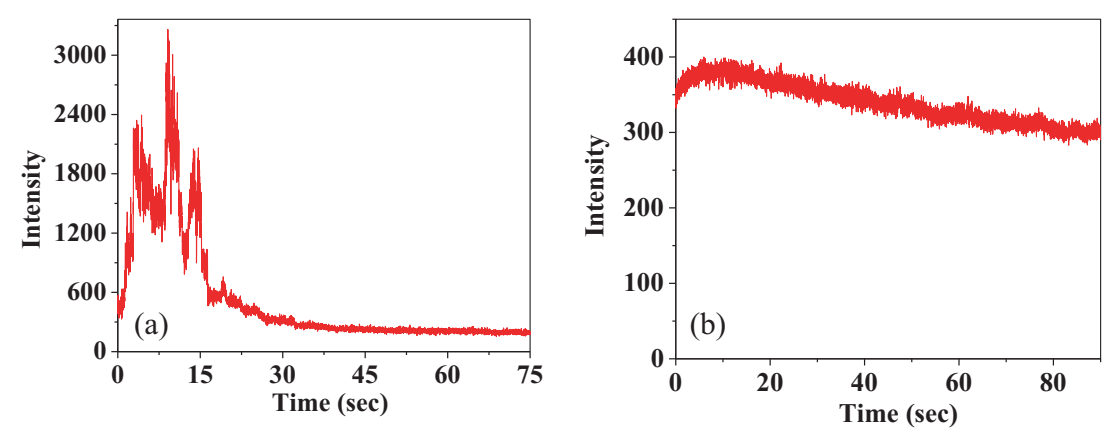

Figure 5. Fluorescence intensity vs. time-trajectories of porphyrin microstructures: (a) TPPS micro-rods and (b) TPPS micro-brush. $\left(\lambda_{\mathrm{ex}}=470 \mathrm{~nm}\right)$. 

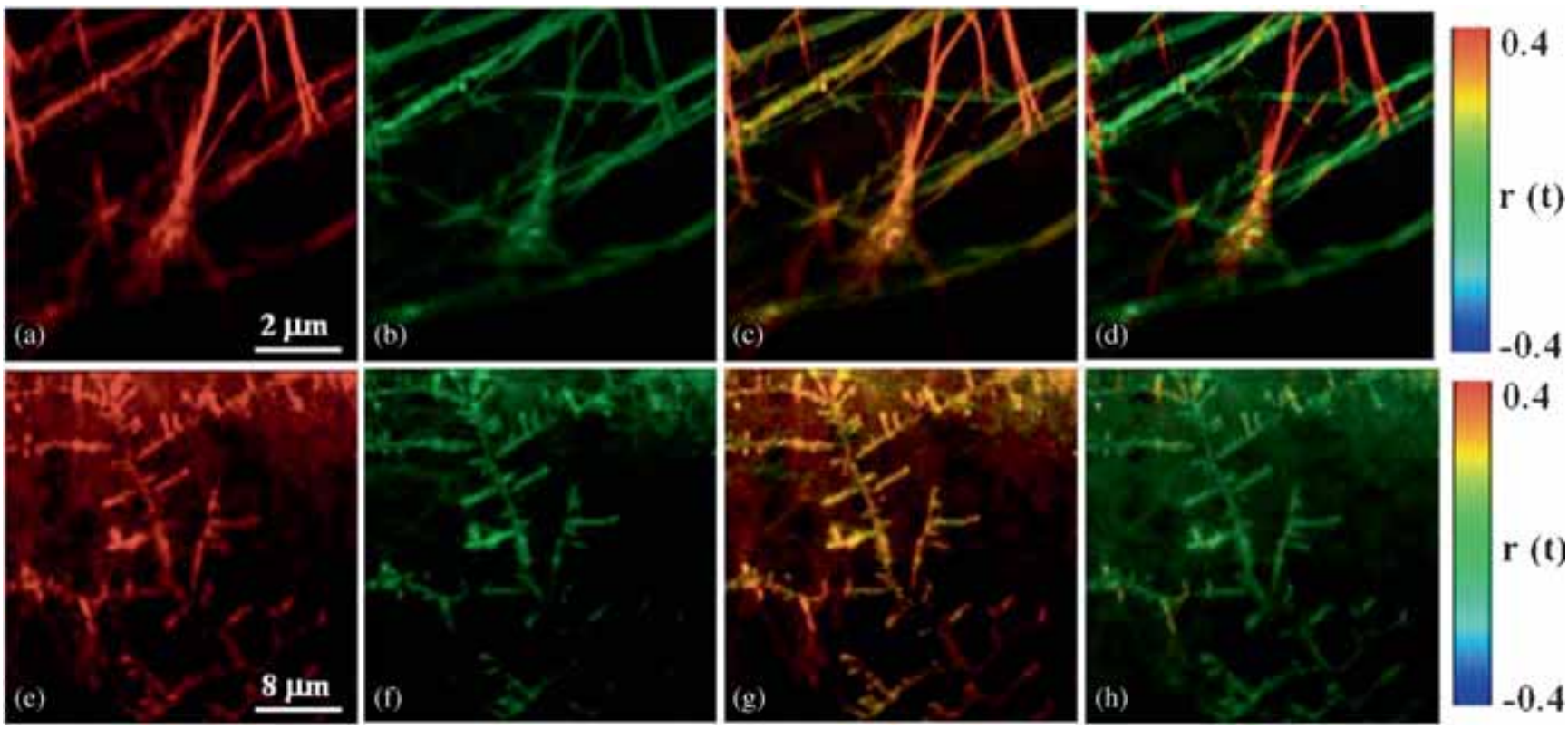

Figure 6. Polarization resolved confocal imaging $\left(\lambda_{\text {ex }}=470 \mathrm{~nm}, \lambda_{\text {em }}=720 \mathrm{~nm}\right.$ ) of TPPS micro-rods (upper panel) and micro-brush (lower panel). Polarization resolved confocal image of micro-rod with (a) parallel component, (b) perpendicular component and (c) merge image. (d) Anisotropy image of micro-rod. Polarization resolved confocal image of micro-brush with (e) parallel component, (f) perpendicular component and (g) merge image. (h) Anisotropy image of porphyrin microbrush. The color bar represents the anisotropy values.
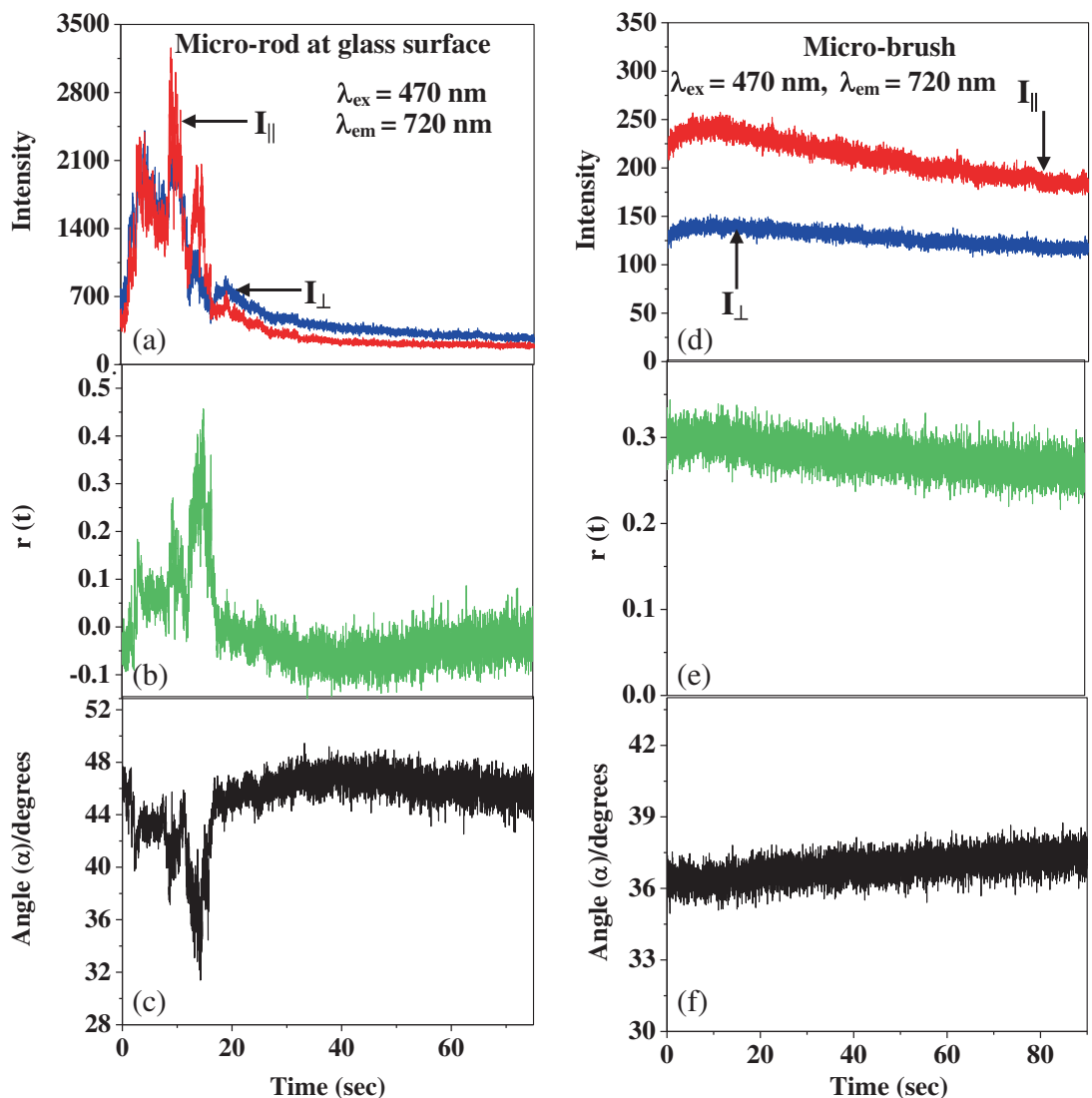

Figure 7. (a, d) Polarization resolved fluorescence intensity vs. time trajectories with parallel (red) and perpendicular (blue) components and (b, e) corresponding anisotropy trace (green) of TPPS micro-rods, micro-brush: at $\lambda_{\mathrm{ex}}=470 \mathrm{~nm}, \lambda_{\mathrm{em}}=720 \mathrm{~nm}$. (c, f) Fluctuation in relative angle $\alpha$ in TPPS micro-rods and micro-brush. 


\subsection{Polarization resolved fluorescence intensity trajectories: TPPS micro-rod and micro-brush}

Figure 7a shows polarization resolved fluorescence intensity trace of TPPS micro-rod at glass surface. The simultaneous monitoring of fluorescence fluctuation in parallel and perpendicular polarization gives real time information about the orientation of the molecular dipoles in such micro-rods. From the trace in Figure 7a we have calculated the anisotropy trace using equation 1.

It is readily seen that $\mathrm{r}(\mathrm{t})$ value fluctuates from -0.1 to 0.4. This indicates reorientation of the transition dipole of TPPS molecule with time in the micro-rod. For the fluctuation in $\mathrm{r}(\mathrm{t})$ value we may compare the relative changes in angle $\alpha$ between the transition dipoles using the formula $\mathrm{r}(\mathrm{t})=\cos (2 \alpha){ }^{35}$ The angle $\alpha$ fluctuates from $30^{\circ}$ to $48^{\circ}$ for porphyrin MR (Figure 7c).

The fluctuation in the high and low anisotropy value suggests that the polarization of the excitons in TPPS micro-rod is less anisotropic and dynamic in nature.

In sharp contrast to the TPPS micro-rod, the microbrush does not exhibit any fluctuation in the anisotropy value (Figure 7e). The anisotropy is fairly constant $\sim 0.25-0.3$. This corresponds to an angle $34^{\circ}-37^{\circ}$ (Figure 7f). No additional fluctuation/transition in anisotropy value has been observed for such micro-brush. This indicates that the TPPS micro-brush is anisotropic and more rigid in nature compared to the TPPS micro-rod.

\section{Conclusions}

This work shows that depending on the rate of evaporation, TPPS forms micro-rods or micro-brushes from an aqueous acidic solution. By focusing laser light at different parts of these micro-rods or micro-brushes we found that fluorescence spectra, exciton dynamics (therefore fluorescence lifetime) and fluorescence anisotropy display spatial inhomogeneity. The presence and absence of discrete fluorescence intensity fluctuations suggest morphology-dependent disordering or ordering of the TPPS units, in the micro-rod and micro-brush, respectively.

\section{Acknowledgements}

Thanks are due to Department of Science and Technology, India (Centre for Ultrafast Spectroscopy and Microscopy Project and J. C. Bose Fellowship) and the Council of Scientific and Industrial Research (CSIR) for generous research support. SC thanks CSIR for awarding fellowships.

\section{References}

1. Lee H, Cheng Y and Fleming G R 2007 Science 316 1462

2. Scholes G D, Fleming G R, Olaya-Castro A and Grondelle R 2011 Nat. Chem. 3763

3. Blankenship R E, Tiede D M, Barber J, Brudvig G W, Fleming G R, Ghirardi M, Gunner M R, Junge W, Kramer D M, Melis A, Moore T A, Moser C C, Nocera D G, Nozik A J, Ort D R, Parson W W, Prince R C and Sayre R T 2011 Science 332805

4. Fleming G R, Schlau-Cohen G S, Amarnath K and Zaks J 2012 Faraday Discuss. 15527

5. Balaban T S 2005 Acc. Chem. Res. 38612

6. Medforth C J, Wang Z, Martin K E, Song Y, Jacobsen $\mathrm{J} \mathrm{L}$ and Shelnutt J 2009 Chem. Commun. 7261

7. McHale J L 2012 J. Phys. Chem. Lett. 3587

8. Verma S and Ghosh H N 2012 J. Phys. Chem. Lett. 3 1877

9. Maiti N C, Ravikanth M, Mazumdar S and Periasamy N 1995 J. Phys. Chem. 9917192

10. Saga $Y$, Wazawa T, Mizoguchi T, Ishii $Y$, Yanagida $T$ and Tamiaki H 2002 Photochem. Photobiol. 75433

11. Roger C, Miloslavina Y, Brunner D, Holzwarth A R and Wurthner F 2008 J. Am. Chem. Soc. 1305929

12. Hajjaj F, Yoon Z S, Yoon M, Park J, Satake A, Kim D and Kobuke Y 2006 J. Am. Chem. Soc. 1284612

13. Rich C C and McHale J L 2012 Phys. Chem. Chem. Phys. 142362

14. Eisele D M, Cone C W, Bloemsma E A, Vlaming S M, van der Kwaak C G F, Silbey R J, Bawendi M G, Knoester J, Rabe J P and Vanden B D A 2012 Nat. Chem. 4655

15. Friesen B A, Nishida K R A, McHale J L and Mazur U 2009 J. Phys. Chem. C 1131709

16. Friesen B A, Wiggins B, McHale J L, Mazur U and Hipps K W 2010 J. Am. Chem. Soc. 1328554

17. Schwab A D, Smith D E, Bond-Watts B, Johnston D E, Hone J, Johnson A T, de Paula J C and Smith W F 2004 Nano. Lett. 41261

18. Kitahama Y, Kimura Y and Takazawa K 2006 Langmuir 227600

19. Hollingsworth J V, Richard A J, Vicente M G H and Russo P S 2012 Biomacromolecules 1360

20. Wan Y, Stradomska A, Fong S, Guo Z, Schaller R D, Wiederrecht G P, Knoester J and Huang L 2014 J. Phys. Chem. C 11824854

21. Ghadamgahi M and Ajloo D 2013 J. Chem. Sci. 125627

22. Setsune J 2012 J. Chem. Sci. 1241151

23. Kalimuthu P, Sivanesan A and John S A 2012 J. Chem. Sci. 1241315

24. Collini E and Scholes G D 2009 Science 323369

25. Cognet L, Tsyboulski D A, Rocha J D R, Doyle C D, Tour J M and Weisman R B 2007 Science 3161465

26. Jendrny M, Aartsma T J and Köhler J 2012 J. Phys. Chem. Lett. 33745

27. Furumaki S, Vacha F, Habuchi S, Tsukatani Y, Bryant D A and Vacha M 2011 J. Am. Chem. Soc. 1336703

28. Shibata $Y$, Tateishi S, Nakabayashi S, Itoh S and Tamiaki H 2010 Biochemistry 497504

29. Sengupta S, Ebeling D, Patwardhan S, Zhang X, Berlepsch H, Bçttcher C, Stepanenko V, Uemura S, Hentschel C, Fuchs H, Grozema F C, Siebbeles L D A, Holzwarth A R, Chi L and Würthner F 2012 Angew. Chem. Int. Ed. $\mathbf{5 1} 6378$ 
30. Forkey J N, Quinlan M E, Shaw M A, Corrie J E T and Goldman Y E 2003 Nature 422399

31. Chappaz-Gillot C, Marek P L, Blaive B J, Canard G, Bürck J, Garab G, Hahn H, Jávorfi T, Kelemen L and Krupke R 2012 J. Am. Chem. Soc. 134 944

32. Furumaki S, Vacha F, Hirata S and Vacha M 2014 ACS Nano. 82176

33. Vallée R A L, Marsal P, Braeken E, Habuchi S, De Schryver F C, Auweraer M V, Beljonne D and Hofkens J 2005 J. Am. Chem. Soc. 12712011

34. Zondervan R, Kulzer F, Berkhout G C G and Orrit M 2007 Proc. Natl. Acad. Sci. USA 104 12628

35. Zhang G, Xiao L, Zhang F, Wang X and Jia S 2010 Phys. Chem. Chem. Phys. 122308

36. Barbara P F, Gesquiere A J, Park S J and Lee Y J 2005 Acc. Chem. Res. 38602
37. Wöll D, Braeken E, Deres A, De Schryver F C, Uji-i H and Hofkens J 2009 Chem. Soc. Rev. 38313

38. Hu D, Yu J, Wong K, Bagchi B, Rossky P J and Barbara P F 2000 Nature 4051030

39. Gradinaru C C, Marushchak D O, Samim M and Krull U J 2010 Analyst 135452

40. Chattoraj S, Chowdhury R, Dey S K, Jana S S and Bhattacharyya K 2015 J. Phys. Chem. B 1198842

41. Chattoraj S and Bhattacharyya K 2014 J. Phys. Chem. C 11822339

42. Drain C M, Varotto A and Radivojevic I 2009 Chem. Rev. 1091630

43. Waltera M G, Wamser C C and Rudineb A B $2010 \mathrm{~J}$. Porphyrins Phthalocyanines $\mathbf{1 4} 759$

44. Miura A, Yanagawa Y and Tamai N 2001 J. Microscopy 202401

45. Leishman C W and McHale J L 2015 J. Phys. Chem. C 11928167 